\title{
Continuous Theta-Burst Stimulation Over the Right Orbitofrontal Cortex in Treatment-Resistant Obsessive-Compulsive Disorder Treatment: A Randomized Sham-Controlled Trial
}

\author{
Weiwei Liu',* \\ Hua Shao $\mathbb{D}^{2, *}$ \\ Jing Liao' \\ Dalu Yang' \\ Maoliang $\mathrm{Ma}^{3}$ \\ Jianli Yang ${ }^{2}$ \\ 'Tianjin Medical University, Tianjin, \\ People's Republic of China; ${ }^{2}$ Clinical \\ Psychology Department, Tianjin Medical \\ University General Hospital, Tianjin, \\ People's Republic of China; ${ }^{3}$ Tianjin \\ Medical University General Hospital \\ Airport Site, Tianjin, People's Republic of \\ China \\ *These authors contributed equally to \\ this work
}

Purpose: Examining whether modulation of right orbitofrontal cortex (OFC) activity by continuous theta-burst stimulation (cTBS) affects obsessive-compulsive disorder (OCD) symptoms.

Patients and Methods: A total of 28 treatment-resistant OCD participants were treated with either active or sham cTBS of the OFC twice per day, for five days a week, for 2 weeks, in a double-blinded manner. Clinical response to treatment was determined using the YaleBrown Obsessive-Compulsive Scale (Y-BOCS).

Results: There were no statistically significant differences between the 2 groups after two weeks of treatment in the Yale-Brown Obsessive-Compulsive Scale score (group*time interaction, F2,20 $=0.996, \mathrm{p}=0.387$ ) and other secondary outcome measures, including anxiety symptoms and responder rates. Depressive symptoms improved significantly in the active group $(\mathrm{p}=0.027)$, but the significant difference disappeared at 6 weeks $(\mathrm{p}=0.089)$.

Conclusion: This is the first randomized controlled study using cTBS in the right OFC to observe the improvement of treatment-resistant OCD symptoms. It is safe to use cTBS, but 2 weeks of treatment is not enough to achieve a curative effect. Future studies are needed to explore more advanced stimulation parameters suitable for the treatment of OCD.

Clinical Trial Registration: www.chictr.org.cn, identifier ChiCTR2000034814.

Keywords: obsessive-compulsive disorder, continuous theta-burst stimulation, orbitofrontal cortex, transcranial magnetic stimulation

\section{Introduction}

Obsessive-compulsive disorder (OCD) is a psychiatric disorder that has a high prevalence. It is characterized by pathological obsessions and compulsions, with a lifetime prevalence of $2.5 \% .^{1}$ Meanwhile, OCD seriously affects patients and their relatives' daily lives, leading to a decline in the quality of life and often accompanied by anxiety and depression. ${ }^{2}$ Serotonin and cognitive-behavioral models are helpful in treatment, but the etiology of OCD remains unknown at present. $^{3}$ Evidence-based treatments for OCD are selective serotonin reuptake inhibitors (SSRIs) and cognitive behavior therapy (CBT). ${ }^{4}$ The combination of pharmacotherapy and CBT in treating OCD has been reported as a more effective strategy than single treatments. ${ }^{5}$ Despite these treatment options, approximately $40 \%$ of patients did not respond to the recommended drugs or CBT. ${ }^{6}$ Therefore,
Correspondence: Jianli Yang

Clinical Psychology Department, Tianjin

Medical University General Hospital,

Anshan Road \#154, Heping District,

Tianjin, People's Republic of China

Email adyy005@163.com 
there is an urgent need to find more safe and effective treatment for OCD. Different add-on stimulation techniques could be effective for patients with severe OCD who were unresponsive to drugs and/or behavioral therapy. $^{7}$

Transcranial magnetic stimulation (TMS) is a form of physical therapy that uses an electric current to create a magnetic field to stimulate specific brain regions by inducing neurophysiological changes. Low-frequency stimulation generally induces lasting suppression of excitability, while high-frequency stimulation generally induces lasting facilitation. ${ }^{8}$ Previous neuropsychological studies have shown that abnormalities of cortical-striatalthalamic-cortical (CSTC) circuits may play a vital role in the pathogenesis of OCD. ${ }^{9}$ TMS can non-invasively and regularly modulate specific brain regions to dissipate symptoms in OCD, and it was relatively safe and welltolerated in clinical practice. Meta-analysis showed that the therapeutic effect of TMS was better than sham TMS in the short term, however, the therapeutic effect is affected by the target, stimulation mode, and intensity. ${ }^{10}$ Most of the previous rTMS studies on OCD use lowfrequency, need more than 20 minutes each time, last about 4-6 weeks, and outpatients need to come to the hospital every day for treatment. The long period of treatment makes patients poor compliance and a high drop-out rate. Previous studies revealed that an accelerated rTMS regimen is a tolerable and noninvasive procedure for shortening the course of treatment and can also improve OCD symptoms within a short period. ${ }^{11-14}$ Theta-burst stimulation (TBS) is a form of TMS, which stimulates the cortex with continuous (cTBS) or intermittent (iTBS) to induce excitement or inhibition. ${ }^{15}$ TBS can be repeated two or more times a day, thus shortening the whole treatment course. At present, most of the studies on TBS in the treatment of OCD are preliminary exploratory studies. cTBS is typically assumed to have long-term depressionlike inhibitory effects that can suppress cortical excitability in targeted regions and can be completed in a short time. ${ }^{16}$ cTBS requires a shorter stimulation duration and lower intensity to induce longer-lasting effects than other rTMS protocols and is also regarded by some authors to be safer than traditional rTMS. ${ }^{17-19}$

At present, the commonly used stimulation targets of OCD are dorsolateral prefrontal cortex(DLPFC),orbitofrontal cortex(OFC), and supplementary motor area(SMA). ${ }^{6}$ The stimulation parameters used for those targets are usually low-frequency stimulation or cTBS, with suppressive effects on cortical excitability. However, studies involving DLPFC and SMA areas had reported inconsistent results. ${ }^{7,18,20}$ Neuroimaging studies have found that the excitability of OFC and other cortical/subcortical structures are increased in patients with $\mathrm{OCD} .^{21}$ Further evidence links the improvement in OCD to decreased hypometabolism in the prefrontal cortex (PFC) and $\mathrm{OFC}^{22}$ Among patients with treatment-resistant $\mathrm{OCD}$, deep brain stimulation (DBS) of the ventral portion of the interior limb of the internal capsule leads to a decrease in overall brain metabolism and, more specifically, in OFC hypermetabolism, after three months of treatment. ${ }^{23}$ Increased resting-state functional connectivity between striatal and prefrontal brain regions (including the OFC and the frontal pole) is closely related to OCD, and the OFC is more accessible to reach than the striatum. ${ }^{24-26}$ Furthermore, TMS targeting the OFC has been found to improve OCD symptoms in patients suffering from drugresistant OCD in some small sample studies. ${ }^{27-29}$ Thus, we speculate that the right OFC may be an important therapeutic target for OCD. Based on evidence reported in previous studies, the present 2-week, double-blind, randomized, sham-controlled trial had the aim to explore the efficacy and safety of cTBS over the right OFC in a sample of treatment-resistant OCD patients.

\section{Patients and Methods Subjects}

The subjects were all OCD patients who came to the outpatient clinic of the Department of Clinical Psychology, General Hospital of Tianjin Medical University from September 2019 to June 2020. Righthanded outpatients aged between 18 and 60 years with DSM-V OCD diagnosed using the Mini-International Neuropsychiatric Interview (MINI) were enrolled in the study. To be eligible, patients had to have a total Y-BOCS score of 16 or more, a total duration of the disease of at least 2 years, and they should have received at least two 12-week adequate sequences and dose of treatment with selective serotonin reuptake inhibitors (SSRIs) but not responding (treatment-resistant). All the psychotropic medications had to be at stable doses for at least 3 months before enrolling in the study, and the current medication regimen (included benzodiazepines) was maintained throughout the treatment and follow-up visits. Exclusion criteria were as follows: diagnosis of other psychiatric disorders (except for depressive or anxious disorders), 
current major depressive disorder (MDD), history of TMS, history of epilepsy or other neurological illness, pregnancy, and any contraindication to TMS. The sample size was estimated using the $G^{*}$ Power 3.1 software. In the previous study ${ }^{28}$ the effect size (Cohen's d value) for the efficacy of rTMS in OCD was 0.44 (which converts to partial eta squared value of 0.05 ). With modest effect size in our study, a power of $95 \%$, alpha of $0.05,2$ groups and 3 repeated measurements, $25 \%$ dropout rate, the proposed study required 18 participants per group. The expected sample size of 36 could not be recruited due to COVID19 restrictions. We enrolled a total of 30 eligible patients, two patients (A man, a woman) withdrew before the trial began due to personal reasons. The rest of the 28 patients have fully understood this study's purpose and steps and signed informed consent forms. A researcher used a random number table, 28 patients were randomly divided into the active group and the sham group, 14 patients in each group. The numbers were written in a sealed Kraft envelope, patients were kept blind to the sequence they were assigned, and the allocation sequence was concealed from recruiters. The envelope was opened immediately before the first session's commencement by the clinician administering the cTBS for each patient.

\section{cTBS Method}

The treatment was administered with the MagPro ${ }^{\circledR}$ X100 with Option stimulator (MagVenture, Inc) using the cool B-65 coil configured inactive or sham mode by flipping the coil over. The stimulus site was Fp2, which corresponds to the right OFC according to the 10-20 International EEG localization system. ${ }^{30}$ The participant's resting motor threshold (RMT) was determined before the first treatment. The RMT was measured as the lowest stimulus intensity capable of eliciting at least 5 motor responses with amplitude of at least $50 \mu \mathrm{V}$ in a series of 10 consecutive trials of single-pulse TMS in the relaxed abductor pollicis brevis muscle. The active treatment group received cTBS at $80 \%$ of RMT, consisting of a burst of three pulses delivered at $50 \mathrm{~Hz}$, repeated every $200 \mathrm{~ms}$ (at $5 \mathrm{~Hz}$ ) for a total of 600 pulses per session. A total of 20 sessions were delivered twice a day (a total of 1200 pulses were delivered per day), with an interval of 15 minutes, 5 days a week, for a total of 2 weeks. The sham treatment group received treatment by flipping the coil over, with identical technical parameters, which induced scalp sensations without penetrating the electric field into the brain. It has a sound and scalp contact similar to those experienced during active stimulation. Adverse events will be carefully monitored during all the study steps by asking patients whether they had experienced any adverse events and the relationship of these events to treatment with TMS.

\section{Outcome Measures}

Subjects were rated at baseline, post-cTBS treatment (2 weeks after baseline, W2), and 4-week follow-up (6 weeks after baseline, W6). They were rated on clinical measures by an independent rater blind to the randomization of TBS groups into active and sham. Our study's severity of obsessions and compulsions was assessed using the Yale-Brown Obsessive-Compulsive Scale (Y-BOCS). Concomitant depressive and anxiety symptoms were assessed using the 17-item Hamilton Depression Rating Scale (HAMD-17) and Hamilton Anxiety Rating Scale (HAMA). Treatment response was defined as a decrease of $25 \%$ on the score than baseline after treatment. ${ }^{31}$

\section{Blind}

In this study, the patients were blind to the arm of treatment, and they were rated on clinical measures by an independent rater who was blind to the randomization of cTBS groups into active and sham. After all treatments, participants were asked to guess whether they had received active or sham stimulation, then they were asked to rate their perceived therapeutic effect on a scale from " 0 " to "9", with " 0 " indicating "not effective at all" and "9" indicating "highly effective". For blinding effectiveness, the self-report ratings were compared using the KruskalWallis Test with group as a factor, and it displayed no significant main effect of group ( $\mathrm{P}=0.987)$.

\section{Statistical Analysis}

Statistical analysis was conducted using SPSS version 24.0 and STATA version 16.0. $\mathrm{X}^{2}$ analysis/fisher exact test was used to compare the groups on categorical variables, and $t$-test was used on continuous variables. Repeated measures analysis of variance was used to compare the primary outcome variable (Y-BOCS score) and other secondary outcome variables (HAMA score and HAMD score) with the scores at 0,2 , and 6 weeks as a withingroup factor and the between-group factor having two active or sham levels cTBS. Statistical testing was twosided, with 0.05 as the criterion for significance. 


\section{Results}

A total of 28 patients with OCD participated in the treatment. They were randomly divided into two groups, of which 2 people dropped out in the active treatment group (one patient could not come to the hospital due to time problems, another due to COVID-19 prevention and control), three patients withdrew during the treatment period in the sham group ( 2 patients due to time problems, one patient did not follow the protocol for personal reasons). Finally, 23 patients were included in the analysis, and the total drop-out rate was $17.9 \%$. There was no significant difference in the drop-out rate between the two groups (Figure 1). During the treatment period, two patients in the treatment group reported scalp pain in the target area, relieved after rest, while the rest had no serious adverse events and no seizures.

The patients' general data are shown in Table 1, and the difference is not statistically significant. The patient maintained the previous drug dose during the treatment period. Among them, $11(91.67 \%)$ in the active group used antidepressants, 2 (16.67\%) used antipsychotics, and $1(8.33 \%)$ used benzodiazepines. In the sham group, 11 (100\%) used antidepressants, $3(27.27 \%)$ used antipsychotics, and 2 (18.18\%) used benzodiazepines. There was no significant difference in drug use between the two groups.

Comparing the two groups' efficacy at different time points is shown in the table below (Table 2). There was no

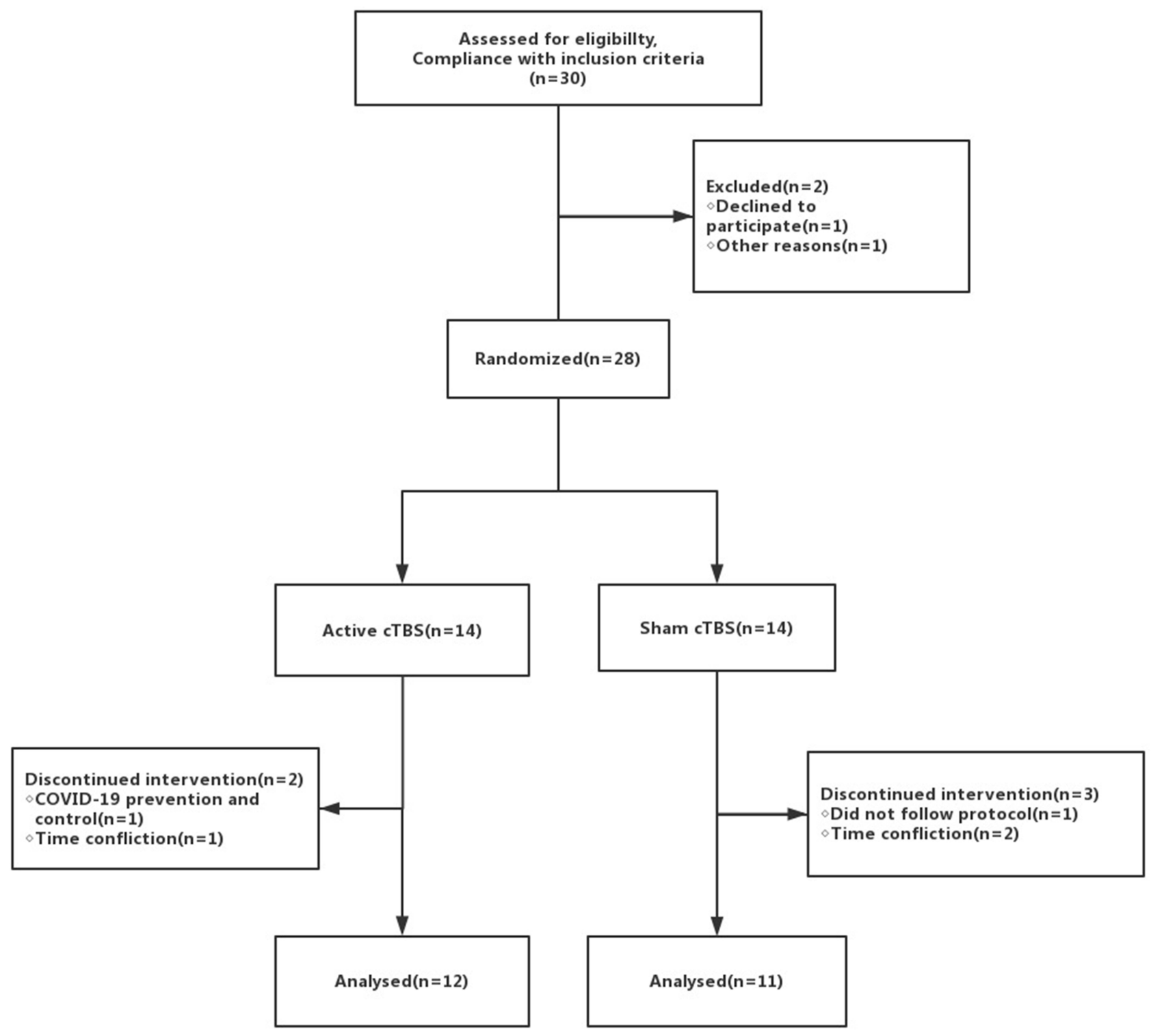

Figure I Consort diagram. 
Table I Baseline Characteristics

\begin{tabular}{|c|c|c|c|}
\hline Variable & $\begin{array}{c}\text { Active Group } \\
\text { (n= I 2) Mean } \\
\text { (SD), } n(\%)\end{array}$ & $\begin{array}{c}\text { Sham Group } \\
\text { (n= II) Mean } \\
\text { (SD), n(\%) }\end{array}$ & $\mathbf{p}$ \\
\hline Age, $y$ & $28.17(9.8 I)$ & $3 I(7.50)$ & 0.296 \\
\hline Sex (male/female) & $5 / 7$ & $5 / 6$ & 1.000 \\
\hline Age at onset, y & $19.92(6.01)$ & $20.72(5.31)$ & $0.24 I$ \\
\hline Duration of illness, $y$ & $8.25(7.66)$ & $10.27(5.10)$ & 0.573 \\
\hline \multicolumn{4}{|l|}{ Medication in use } \\
\hline Antidepressant & II(9I.67) & $\mathrm{II}(100)$ & 1.000 \\
\hline Antipsychotics & $2(16.67)$ & $3(27.27)$ & 0.640 \\
\hline Benzodiazepine & I(8.33) & $2(18.18)$ & 0.590 \\
\hline Y-BOCS & $26.33(2.8 I)$ & $28.27(5.00)$ & 0.275 \\
\hline HAMA & $10.08(5.47)$ & $10.91(6.99)$ & 0.754 \\
\hline HAMD & |3.58(6.37) & $9.45(4.99)$ & 0.100 \\
\hline
\end{tabular}

significant difference in the Y-BOCS score between the active and sham groups at the baseline $(p=0.275)$. Depending on different time points, the Y-BOCS score of the two groups decreased. Compared with the baseline, there was a significant difference $(p=0.021)$, but there was no statistical difference between the two groups $(p=0.387)$. Although there was no group $*$ time interaction $(\mathrm{F}=0.584, \mathrm{P}=0.567)$, the repeated measures analyses indicated that the HAMA score decreasing significantly compared with the baseline $(\mathrm{F}=9.509, \mathrm{P}=0.001)$. Besides, there were significant differences in HAMD scores per time, group $(\mathrm{F}=16.544, \mathrm{P}<0.001)$, and the group * time interaction $(\mathrm{F}=4.132, \mathrm{P}=0.031)$.

We compared the effective rate of the two groups, the scale score reduced by $25 \%$ as applicable, and the total effective rate $=$ improve number/total number of patients The three outcome indicators calculated the effective rate and carried out the chi-square test and fisher exact probability test, as detailed in Table 3. There was only a significant difference in the effective rate between the two groups in the depression score after two weeks $(p=0.027)$, consistent with our previous results.

Figure 2 shows the mean Y-BOCS scores of the active and sham groups at different time points, and there is no significant difference between the two groups ( $\mathrm{P}>0.05)$.

There was no significant difference in HAMA and HAMD scores at baseline. With the prolongation of treatment time, HAMA scores decreased significantly after two weeks of treatment ( $\mathrm{p}=0.001)$, but there was no statistical difference between the two groups $(\mathrm{p}=0.567)$. The changing trend of HAMA scores is shown in Figure 3. Within two weeks, the decrease of HAMD score in the active group was more evident than that in the sham group, the decrease of HAMD score in the two groups was statistically significant, and the changing trend of the HAMD score was shown in Figure 4.

\section{Discussion}

The efficacy studies of TMS in OCD, including open trials, sham-controlled studies and comparison studies, have involved different methodological designs using various stimulation parameters (eg, frequency, intensity, stimulation site, number of pulse, duration of treatment). ${ }^{32}$ In our study, the treatment option and target selection were innovative. To the best of our knowledge, it was the first randomized, double-blind study on the efficacy of cTBS

Table 2 Clinical Outcome

\begin{tabular}{|c|c|c|c|c|c|c|}
\hline \multirow[t]{2}{*}{ Scale } & \multirow[t]{2}{*}{ Active, Mean(SD) } & \multirow[t]{2}{*}{ Sham, Mean (SD) } & \multicolumn{2}{|c|}{ ANOVA Time Effect } & \multicolumn{2}{|c|}{ ANOVA Group*Time Interaction } \\
\hline & & & $\mathbf{F}$ & $\mathbf{p}$ & $\mathbf{F}$ & $\mathbf{p}$ \\
\hline \multicolumn{7}{|l|}{ Y-BOCS } \\
\hline Week 0 & $26.33(2.8 I)$ & $28.27(5.00)$ & $* F 2,20=4.743$ & 0.021 & $* F 2,20=0.996$ & 0.387 \\
\hline Week 2 & $24.75(2.38)$ & $25.09(4.32)$ & & & & \\
\hline Week 6 & 23.50(3.97) & $24.82(3.82)$ & & & & \\
\hline \multicolumn{7}{|l|}{ HAMD-I7 } \\
\hline Week 0 & $13.58(6.37)$ & $9.45(4.99)$ & $* \mathrm{~F} 2,20=16.544$ & $<0.001$ & $* F 2,20=4.132$ & 0.031 \\
\hline Week 2 & $6.42(4.58)$ & $7.09(2.21)$ & & & & \\
\hline Week 6 & $5.17(2.66)$ & $6.64(2.50)$ & & & & \\
\hline \multicolumn{7}{|l|}{ HAMA } \\
\hline Week 0 & $10.08(5.47)$ & $10.91(6.99)$ & $* \mathrm{~F} 2,20=9.509$ & 0.001 & ${ }^{*} \mathrm{~F} 2,20=0.584$ & 0.567 \\
\hline Week 2 & $7.17(2.82)$ & $8.36(4.99)$ & & & & \\
\hline Week 6 & $6.08(2.91)$ & $8.00(4.56)$ & & & & \\
\hline
\end{tabular}

Note: *Correction for nonsphericity.

Abbreviation: ANOVA, analysis of variance. 
Table 3 Comparison of the Effective Rate After Treatment in Active and Sham Groups

\begin{tabular}{|l|r|r|r|r|r|}
\hline & Time & Active (\%) & Sham (\%) & $\boldsymbol{x}^{2}$ & $\mathbf{p}$ \\
\hline Y-BOCS & $2 \mathrm{w}$ & 16.67 & 9.09 & 0.290 & 1.000 \\
& 6w & 25 & 18.18 & 0.157 & 1.000 \\
\hline HAMA & $2 \mathrm{w}$ & 41.67 & 45.45 & 0.034 & 1.000 \\
& $6 \mathrm{w}$ & 58.33 & 63.64 & 0.068 & 1.000 \\
\hline HAMD & $2 \mathrm{w}$ & 91.67 & 45.45 & 5.789 & 0.027 \\
& $6 \mathrm{w}$ & 83.33 & 45.45 & 3.630 & 0.089 \\
\hline
\end{tabular}

over the right $\mathrm{OFC}$ in treatment-resistant OCD patients. Both groups improved significantly in depression and anxiety, and the Y-BOCS score decreased significantly after two weeks of treatment. However, except for the HAMD score, there was no significant difference in Y-BOCS and HAMA scores between the active and sham groups. Previous studies demonstrated that TBS could induce a more prominent and longer-lasting effect on cortical excitability than standard rTMS. ${ }^{33,34}$ Among the published randomized-controlled trials, only two have reported cTBS in treatment-resistant $\mathrm{OCD}$, and the results showed that cTBS was not effective in treating obsessive symptoms, which was consistent with ours. ${ }^{17,18}$
Studies in refractory depression have found that accelerated rTMS could reduce treatment response time. ${ }^{35}$ A previous open-label study found that low-frequency rTMS twice a day could improve the symptoms of treatment-resistant OCD in 10 days, while traditional rTMS needed at least four weeks. ${ }^{36}$ Neuroimaging studies had shown that accelerated rTMS requires at least a 10-minute interval, otherwise, the additional neuroplasticity effects may not occur. ${ }^{37}$ However, the optimal time interval is still a topic to be researched through clinical trials. In our study, 15-minute intervals were set during the twicedaily cTBS protocol. Among all the patients who completed the treatment, two in the active group reported scalp pain in the target area, but the pain was relieved after rest, and no serious adverse events occurred, indicating that the twice-daily cTBS regimen is safe and well-tolerated.

According to current neurobiological models, the OFC is one of the core features in the pathophysiology of OCD. Effective treatments for OCD lead to a decrease in the hypermetabolism observed in the OFC. ${ }^{22}$ The previous investigation in OCD patients showed reduced functional connectivity in the right DLPFC and the right OFC, and activity in the right OFC had an inhibitory on the right DLPFC. ${ }^{38}$ There have been previous studies on the left,

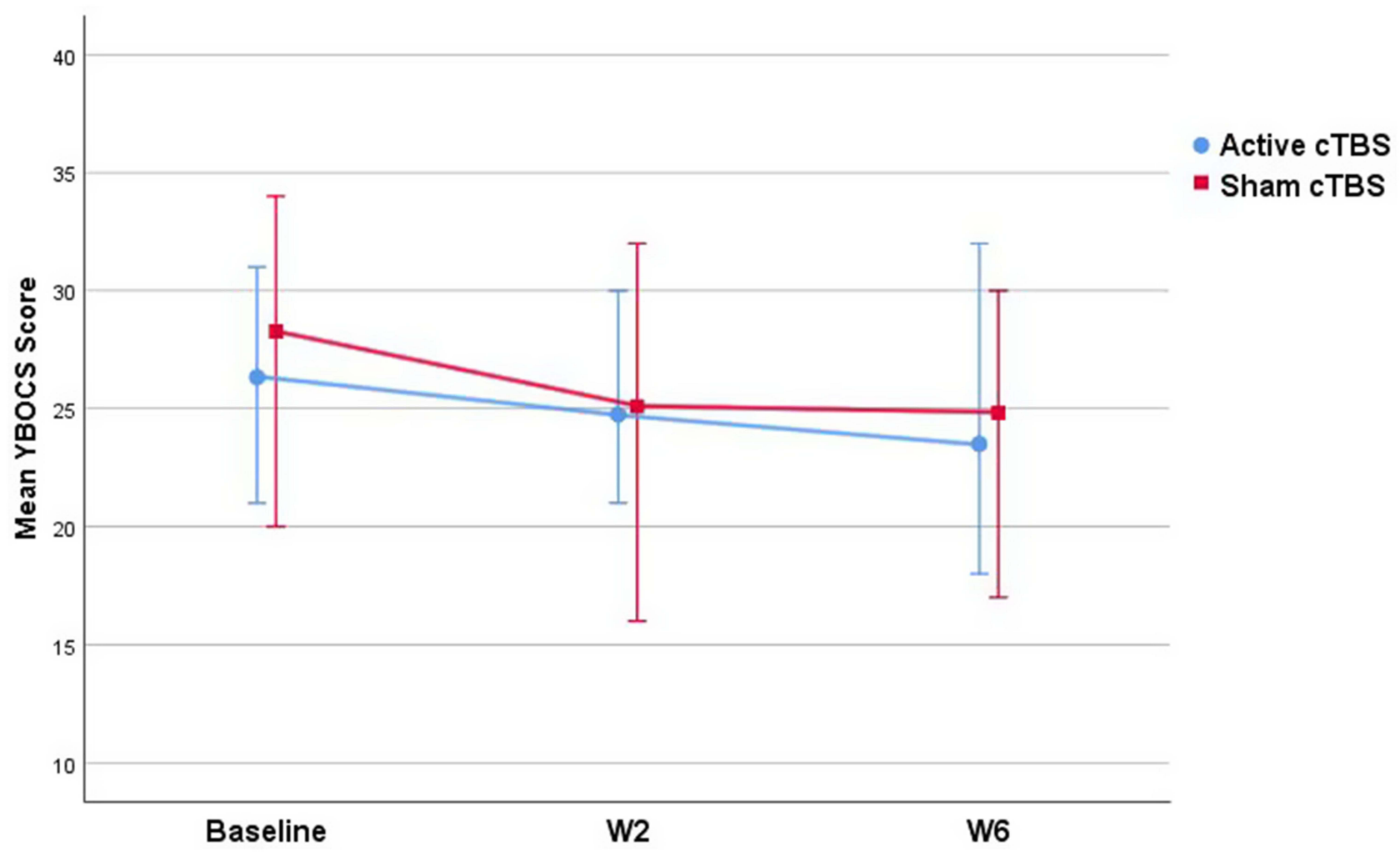

Figure 2 Y-BOCS scores in active and sham cTBS groups. 


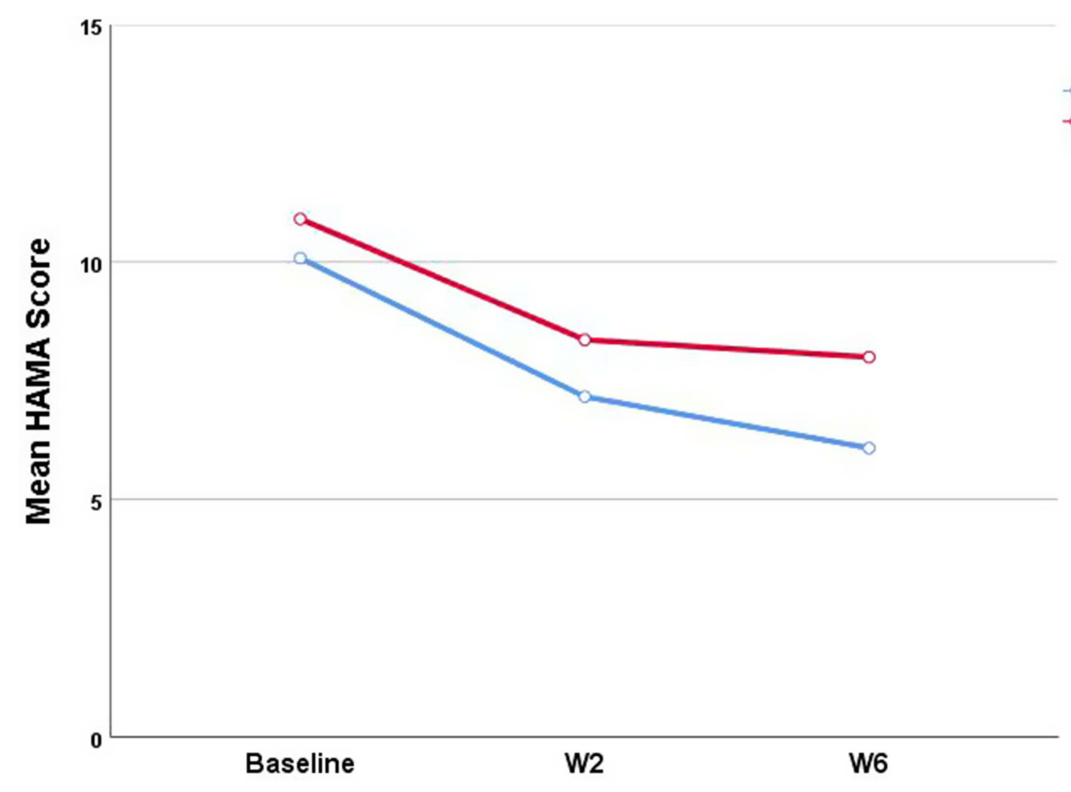

Figure 3 Comparison of mean HAMA scores in active and sham groups.

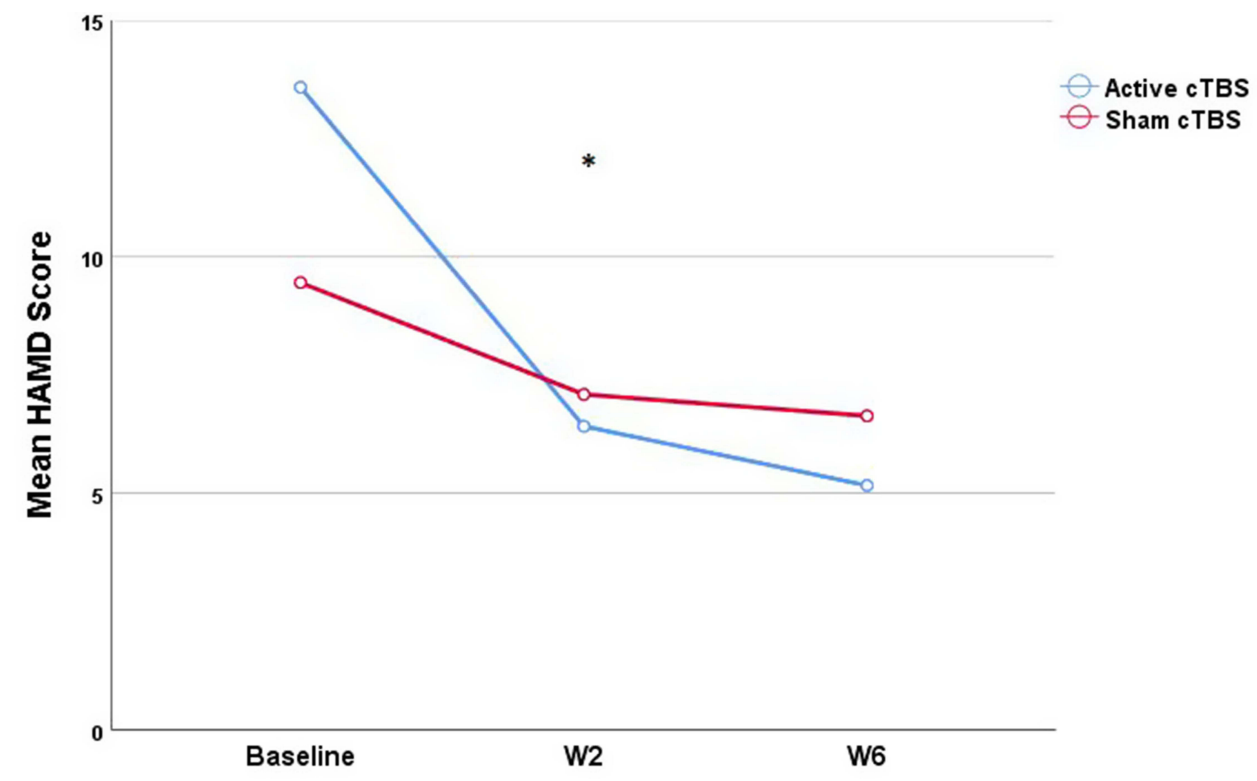

Figure 4 Comparison of mean HAMD scores in active and sham groups. ${ }^{*} p<0.05$

right, and bilateral OFC, and the results are not consistent because of the different protocols used in treatment. ${ }^{27,39-42}$ A double-blind cross-over study evaluated the efficacy of low-frequency rTMS over the right OFC with a doublecone coil in patients suffering from OCD. The study observed a significant decrease from baseline in the Y-BOCS scores after both active and sham stimulation. This decrease tended to be more prominent after active stimulation than after sham stimulation. Active versus sham PET scan contrasts showed that stimulation was related to a bilateral decrease in the metabolism of the OFC. ${ }^{29}$ We selected the less studied right OFC as the target area, which might be a more theoretically appropriate target based on the previous study. The stimulus intensity of the traditional rTMS regimen ranges from $80 \%$ to $120 \%$ of RMT, and we used $80 \%$ of RMT in the trial, which is consistent with the safety intensity used in previous studies by Huang et al. ${ }^{15} \mathrm{~A}$ randomized noninferiority trial found that high-intensity iTBS $(120 \%$ RMT) exhibited the same efficacy as traditional rTMS 
protocols in patients with depression and were well tolerated. ${ }^{43}$ However, there is no evidence that higher intensity of cTBS is more effective in treating OCD, which may serve as a direction for future research.

Patients with MDD and/or suicidal ideation were excluded from the study. There was no significant difference in the HAMD scores between the two groups before treatment, and depressive symptoms were significantly relieved after treatment (the score decreased by $25 \%$ was defined as treatment response). Previous studies have found that the marginal structure of the frontal lobestriatum-thalamic emotional loop is abnormal in MDD patients. ${ }^{44}$ We speculate that this loop may also be activated when we stimulate the OFC, leading to the improvement of depression symptoms. There were significant differences in time for depressive symptoms of the active group, and these were significantly lower at W2 but not at W6, suggesting that the effects may be short-term. A cross-sectional study showed that public anxiety, depression, and psychological disturbances were widespread, and symptoms significantly increase during the current COVID-19 pandemic. ${ }^{45}$ Patients were exposed to a continuous social stressor (COVID-19) during the trial, resulting in impaired therapeutic effectiveness. In addition, the baseline depressive symptoms were low and may thus be subsyndromal. It may be possible to conduct a larger randomized controlled trial in the future.

The failure of our trial may can be explained by the following aspects: a. This study's objects are treatmentresistant OCD patients whose treatment is difficult due to early-onset and a prolonged disease course; ${ }^{46} \mathrm{~b}$. Although we have increased the frequency of treatments per day, the 2-week treatment cycle may not be sufficient to cause significant and continuous changes in OCD symptoms; c. The intensity of stimulation is also an essential factor affecting the efficacy. It is not clear whether increasing the intensity of stimulation can enhance the efficacy. This study chose a stimulus intensity of $80 \%$ RMT used in most cTBS studies, but it may be somewhat insufficient in treatment-resistant OCD; d. We locate the OFC target convenient and straightforward through the international 10-20 EEG system, and this method is not as accurate as neuronavigation because of each person's brain's anatomical differences; e. The sample size of this study was small, and there may be high individual differences between responses to noninvasive brain stimulation regimens, including routine rTMS and TBS; ${ }^{47}$ f. We used was a commonly used figure-of- 8 shaped coil, which was also used in the studies of Dutta et al and Harika-
Germaneau et al, ${ }^{17,18}$ but did not achieve clinical efficacy. It may be due to the shallow penetration depth of the magnetic field, which cannot reach the depth of the target stimulation. A study by Carmi et al showed that high-frequency dTMS on the MPFC and ACC could significantly improve OCD symptoms. They used a special H-coil that allows magnetic stimulation to penetrate the brain more deeply and extensively, thus achieving therapeutic effects. ${ }^{48}$ Although the dTMS study showed more promising results, there are several methodological differences (such as coil type, sample size, and symptom provocation before TMS application), and further research is needed in the future.

\section{Conclusion}

The results of this study suggest that two weeks of cTBS may not be optimal for improving OCD symptoms in treatment-resistant OCD, but twice-daily cTBS for OCD patients is safe, well-tolerated, without apparent side effects. Future studies should recruit a larger sample size and investigate whether treatment duration should be extended to facilitate the maintenance of treatment gains.

\section{Data Sharing Statement}

The datasets generated during and/or analyzed during the current study are available from the corresponding author on reasonable request.

\section{Ethics Statement}

This study has been approved by the appropriate ethics committee of Tianjin Medical University General Hospital and performed according to the ethical standards laid down in the 1964 Declaration of Helsinki and its later amendments. Informed consent was obtained from all individual participants included in the study.

\section{Acknowledgments}

We would like to thank all participants who joined this study. Weiwei Liu and Hua Shao are shared first authors.

\section{Funding}

There was no financial support for the research and publication of this article.

\section{Disclosure}

The authors report no conflicts of interest in this work. 


\section{References}

1. Ruscio AM, Stein DJ, Chiu WT, Kessler RC. The epidemiology of obsessive-compulsive disorder in the National Comorbidity Survey Replication. Mol Psychiatry. 2010;15(1):53-63. doi:10.1038/ mp.2008.94

2. Macy AS, Theo JN, Kaufmann SCV, et al. Quality of life in obsessive compulsive disorder. CNS Spectr. 2013;18(1):21-33. doi:10.1017/ S1092852912000697

3. Stein DJ, Costa DLC, Lochner C, et al. Obsessive-compulsive disorder. Nat Rev Dis Prim. 2019;5(1):52. doi:10.1038/s41572-0190102-3

4. Bandelow B, Zohar J, Hollander E, Kasper S, Möller H-J. WFSBP task force on treatment guidelines for anxiety, obsessive-compulsive and post-traumatic stress disorders. World Federation of Societies of Biological Psychiatry (WFSBP) guidelines for the pharmacological treatment of anxiety, obsessive-compulsive and post-traumatic stress disorders-first revision. World J Biol Psychiatry. 2008;9 (4):248-312. doi:10.1080/15622970802465807

5. Meng FQ, Han HY, Luo J, et al. Efficacy of cognitive behavioural therapy with medication for patients with obsessive-compulsive disorder: a multicentre randomised controlled trial in China. $J$ Affect Disord. 2019;253:184-192. doi:10.1016/j.jad.2019.04.090

6. Shivakumar V, Dinakaran D, Narayanaswamy JC, Venkatasubramanian G. Noninvasive brain stimulation in obsessive-compulsive disorder. Indian J Psychiatry. 2019;61(Suppl 1):S66-S76. doi:10.4103/psychiatry.IndianJPsychiatry_522_18

7. Rapinesi C, Kotzalidis GD, Ferracuti S, Sani G, Girardi P, Del Casale A. Brain stimulation in obsessive-compulsive disorder (OCD): a systematic review. Curr Neuropharmacol. 2019;17 (8):787-807. doi:10.2174/1570159X17666190409142555

8. Houdayer E, Degardin A, Cassim F, Bocquillon P, Derambure P, Devanne $\mathrm{H}$. The effects of low- and high-frequency repetitive TMS on the input/output properties of the human corticospinal pathway. Exp Brain Res. 2008;187(2):207-217. doi:10.1007/s00221-008-1294-z

9. Tang W, Zhu Q, Gong X, Zhu C, Wang Y, Chen S. Cortico-striatothalamo-cortical circuit abnormalities in obsessive-compulsive disorder: a voxel-based morphometric and fMRI study of the whole brain. Behav Brain Res. 2016;313:17-22. doi:10.1016/j.bbr.2016.07.004

10. Zhou DD, Wang W, Wang GM, Li DQ, Kuang L. An updated meta-analysis: short-term therapeutic effects of repeated transcranial magnetic stimulation in treating obsessive-compulsive disorder J Affect Disord. 2017;215:187-196. doi:10.1016/j.jad.2017.03.033

11. Modirrousta M, Meek BP, Wikstrom SL. Efficacy of twice-daily vs once-daily sessions of repetitive transcranial magnetic stimulation in the treatment of major depressive disorder: a retrospective study. Neuropsychiatr Dis Treat. 2018;14:309-316. doi:10.2147/NDT. S151841

12. Zhao D, Li Y, Liu T, Voon V, Yuan TF. Twice-daily theta burst stimulation of the dorsolateral prefrontal cortex reduces methamphetamine craving: a pilot study. Front Neurosci. 2020;14:208 doi:10.3389/fnins.2020.00208

13. Schulze L, Feffer K, Lozano C, et al. Number of pulses or number of sessions? An open-label study of trajectories of improvement for once-vs. twice-daily dorsomedial prefrontal rTMS in major depression. Brain Stimul. 2018;11(2):327-336. doi:10.1016/j. brs.2017.11.002

14. Williams NR, Sudheimer KD, Cole EJ, et al. Accelerated neuromodulation therapy for obsessive-compulsive disorder. Brain Stimul. 2021;14(2):435-437. doi:10.1016/j.brs.2021.02.013

15. Huang YZ, Edwards MJ, Rounis E, Bhatia KP, Rothwell JC. Theta burst stimulation of the human motor cortex. Neuron. 2005;45 (2):201-206. doi:10.1016/j.neuron.2004.12.033
16. Vernet M, Bashir S, Yoo WK, et al. Reproducibility of the effects of theta burst stimulation on motor cortical plasticity in healthy participants. Clin Neurophysiol. 2014;125(2):320-326. doi:10.1016/ j.clinph.2013.07.004

17. Dutta P, Dhyani M, Garg S, et al. Efficacy of intensive orbitofrontal continuous theta burst stimulation (iOFcTBS) in obsessive compulsive disorder: a randomized placebo controlled study. Psychiatry Res. 2021;298:113784. doi:10.1016/j.psychres.2021.113784

18. Harika-Germaneau G, Rachid F, Chatard A, et al. Continuous theta burst stimulation over the supplementary motor area in refractory obsessive-compulsive disorder treatment: a randomized sham-controlled trial. Brain Stimul. 2019;12(6):1565-1571. doi:10.1016/j.brs.2019.07.019

19. Doeltgen SH, Ridding MC. Modulation of cortical motor networks following primed $\theta$ burst transcranial magnetic stimulation. Exp Brain Res. 2011;215(3-4):199-206. doi:10.1007/s00221-011-2886-6

20. Ma X, Huang Y, Liao L, Jin Y. A randomized double-blinded shamcontrolled trial of $\alpha$ electroencephalogram-guided transcranial magnetic stimulation for obsessive-compulsive disorder. Chin Med J. 2014;127(4):601-606.

21. Lagemann T, Rentzsch J, Montag C, et al. Early orbitofrontal hyperactivation in obsessive-compulsive disorder. Psychiatry Res. 2012;202(3):257-263. doi:10.1016/j.pscychresns.2011.10.002

22. Nakao T, Nakagawa A, Yoshiura T, et al. Brain activation of patients with obsessive-compulsive disorder during neuropsychological and symptom provocation tasks before and after symptom improvement: a functional magnetic resonance imaging study. Biol Psychiatry. 2005;57(8):901-910. doi:10.1016/j.biopsych.2004.12.039

23. Nuttin BJ, Gabriëls LA, Cosyns PR, et al. Long-term electrical capsular stimulation in patients with obsessive-compulsive disorder. Neurosurgery. 2003;52(6):1263-1272. doi:10.1227/01.NEU.0000064565.49299.9A

24. Harrison BJ, Pujol J, Cardoner N, et al. Brain corticostriatal systems and the major clinical symptom dimensions of obsessive-compulsive disorder. Biol Psychiatry. 2013;73(4):321-328. doi:10.1016/j. biopsych.2012.10.006

25. Hou JM, Zhao M, Zhang W, et al. Resting-state functional connectivity abnormalities in patients with obsessive-compulsive disorder and their healthy first-degree relatives. $J$ Psychiatry Neurosci. 2014;39(5):304-311. doi:10.1503/jpn.130220

26. Cocchi L, Zalesky A, Nott Z, Whybird G, Fitzgerald PB, Breakspear M. Transcranial magnetic stimulation in obsessive-compulsive disorder: a focus on network mechanisms and state dependence. Neuroimage Clin. 2018;19:661-674. doi:10.1016/j.nicl.2018.05.029

27. Kumar S, Singh S, Chadda RK, Verma R, Kumar N. The effect of low-frequency repetitive transcranial magnetic stimulation at orbitofrontal cortex in the treatment of patients with medication-refractory obsessive-compulsive disorder: a retrospective open study. $J$ Ect. 2018;34(2):e16-e19. doi:10.1097/YCT.0000000000000462

28. Ruffini C, Locatelli M, Lucca A, Benedetti F, Insacco C, Smeraldi E. Augmentation effect of repetitive transcranial magnetic stimulation over the orbitofrontal cortex in drug-resistant obsessive-compulsive disorder patients: a controlled investigation. Prim Care Companion J Clin Psychiatry. 2009;11(5):226-230. doi:10.4088/PCC.08m00663

29. Nauczyciel C, Le Jeune F, Naudet F, et al. Repetitive transcranial magnetic stimulation over the orbitofrontal cortex for obsessive-compulsive disorder: a double-blind, crossover study. Transl Psychiatry. 2014;4(9):e436. doi:10.1038/tp.2014.62

30. Wang J, You D, Wang H, et al. Association between homocysteine and obesity: a meta-analysis. J Evid Based Med. 2020. doi:10.1111/ jebm. 12412

31. Pallanti S, Hollander E, Bienstock C, et al. Treatment non-response in OCD: methodological issues and operational definitions. Int $J$ Neuropsychopharmacol. 2002;5(2):181-191. doi:10.1017/ S1461145702002900 
32. Trevizol AP, Shiozawa P, Cook IA, et al. Transcranial magnetic stimulation for obsessive-compulsive disorder: an updated systematic review and meta-analysis. J ECT. 2016;32(4):262-266. doi:10.1097/ YCT.0000000000000335

33. Suppa A, Huang YZ, Funke K, et al. Ten years of theta burst stimulation in humans: established knowledge, unknowns and prospects. Brain Stimul. 2016;9(3):323-335. doi:10.1016/j. brs.2016.01.006

34. Rounis E, Huang YZ. Theta burst stimulation in humans: a need for better understanding effects of brain stimulation in health and disease. Exp Brain Res. 2020;238(7-8):1707-1714. doi:10.1007/ s00221-020-05880-1

35. Baeken C. Accelerated rTMS: a potential treatment to alleviate refractory depression. Front Psychol. 2018;9:2017. doi:10.3389/ fpsyg.2018.02017

36. Tandt HLN, Van de Velde N, De Witte S, Audenaert K, Baeken C, Lemmens GMD. Is twice daily LF-rTMS a viable treatment option for treatment-resistant OCD? Results from an open-label feasibility study. Eur Arch Psychiatry Clin Neurosci. 2021;271(1):211-214.

37. Nettekoven C, Volz LJ, Leimbach M, et al. Inter-individual variability in cortical excitability and motor network connectivity following multiple blocks of rTMS. NeuroImage. 2015;118:209-218.

38. Li H, Hu X, Gao Y, et al. Neural primacy of the dorsolateral prefrontal cortex in patients with obsessive-compulsive disorder. Neuroimage Clin. 2020;28:102432.

39. Maatoug R, Ekmen A, Valero-Cabre A, Millet B. Stimulation therapeutic approaches to better understand obsessive compulsive disorder: the issue of 'where' to treat. Encephale. 2020;46(5):399-403. doi:10.1016/j.encep.2019.11.003

40. Senova S, Clair AH, Palfi S, Yelnik J, Domenech P, Mallet L. Deep brain stimulation for refractory obsessive-compulsive disorder: towards an individualized approach. Front Psychiatry. 2019;10:905. doi:10.3389/fpsyt.2019.00905
41. Singh S, Kumar S, Gupta A, Verma R, Kumar N. Effectiveness and predictors of response to $1-\mathrm{Hz}$ repetitive transcranial magnetic stimulation in patients with obsessive-compulsive disorder. J Ect. 2019;35 (1):61-66. doi:10.1097/YCT.0000000000000530

42. Mondino M, Haesebaert F, Poulet E, Saoud M, Brunelin J. Efficacy of cathodal transcranial direct current stimulation over the left orbitofrontal cortex in a patient with treatment-resistant obsessive-compulsive disorder. $J$ Ect. 2015;31(4):271-272. doi:10.1097/YCT.0000000000000218

43. Blumberger DM, Vila-Rodriguez F, Thorpe KE, et al. Effectiveness of theta burst versus high-frequency repetitive transcranial magnetic stimulation in patients with depression (THREE-D): a randomised non-inferiority trial. Lancet. 2018;391(10131):1683-1692. doi:10.1016/S0140-6736(18)30295-2

44. Lai CH. Promising neuroimaging biomarkers in depression. Psychiatry Investig. 2019;16(9):662-670. doi:10.30773/ pi.2019.07.25.2

45. Bäuerle A, Teufel M, Musche V, et al. Increased generalized anxiety, depression and distress during the COVID-19 pandemic: a cross-sectional study in Germany. $J$ Public Health. 2020;42 (4):672-678. doi:10.1093/pubmed/fdaa106

46. Jakubovski E, Diniz JB, Valerio C, et al. Clinical predictors of long-term outcome in obsessive-compulsive disorder. Depress Anxiety. 2013;30(8):763-772. doi:10.1002/da.22013

47. López-Alonso V, Cheeran B, Río-Rodríguez D, Fernández-del-Olmo M. Inter-individual variability in response to non-invasive brain stimulation paradigms. Brain Stimul. 2014;7(3):372-380. doi:10.1016/j.brs.2014.02.004

48. Carmi L, Tendler A, Bystritsky A, et al. Efficacy and safety of deep transcranial magnetic stimulation for obsessive-compulsive disorder: a prospective multicenter randomized double-blind placebo-controlled trial. Am J Psychiatry. 2019;176(11):931-938. doi:10.1176/appi.ajp.2019.18101180
International Journal of General Medicine

\section{Publish your work in this journal}

The International Journal of General Medicine is an international, peer-reviewed open-access journal that focuses on general and internal medicine, pathogenesis, epidemiology, diagnosis, monitoring and treatment protocols. The journal is characterized by the rapid reporting of reviews, original research and clinical studies across all disease areas. The manuscript management system is completely online and includes a very quick and fair peer-review system, which is all easy to use. Visit http://www.dovepress.com/ testimonials.php to read real quotes from published authors. 